\title{
Contribución a la Caracterización y Evaluación de la Actividad Antioxidante de las Antocianinas del Fruto de Ivilan (Monnina Obtusifolia H.B.K)
}

\author{
Nelson H. Hurtado y Claudia Charfuelan \\ Universidad de Nariño, Departamento de Química, Grupo de Investigación en Productos de Importancia Biológica \\ (GIPIB), Calle 18 Carrera 50, Campus Torobajo, San Juan de Pasto - Nariño - Colombia. \\ (e-mail: nhurtado@udenar.edu.co, claudiach1129@gmail.com)
}

Recibido Nov. 29, 2018; Aceptado Ene. 29, 2019; Versión final Mar. 5, 2019, Publicado Oct. 2019

\begin{abstract}
Resumen
Mediante el uso de diferentes técnicas cromatográficas se obtuvieron extractos ricos en polifenoles (antocianinas). A partir de los extractos purificados se identificaron por primera vez las antocianinas mayoritarias del fruto de Monnina Obtusifolia: una piroantocianina y tres antocianinas derivadas de delfinidina, cianidina y peonidina. Se evaluó además la contribución relativa de diferentes tipos de fracciones aisladas del fruto (extractos crudos, purificados y compuestos puros) sobre sus propiedades antioxidantes. La antocianina con mayor poder para capturar el radical sintético $\mathrm{ABTS}^{*}+$ fue el compuesto derivado de delfinidina. Se encontró que las agliconas con un solo un grupo $\mathrm{OH}$ en el anillo $\mathrm{B}\left(4^{\prime}-\mathrm{OH}\right)$ presentan menor actividad antioxidante, comparado con compuestos que poseen agrupación $3^{\prime}, 4^{\prime}$ di-OH sustituidos como los derivados de delfinidina y cianidina. El análisis de la capacidad reductora de Folin-Ciocalteau y la capacidad antioxidante equivalente al trolox de las fracciones aisladas sugieren que el fruto de Monnina Obtusifolia puede ser considerado como una fuente importante de compuestos bioactivos, para su uso en la industria.
\end{abstract}

\section{Contribution to the Characterization and Evaluation of the Antioxidant Activity of Anthocyanis of Ivilan Fruit (Monnina Obtusifolia H.B.K)}

\begin{abstract}
Through the use of different chromatographic techniques extracts rich in polyphenols (anthocyanins) were obtained. From the purified extracts, the main anthocyanins of the Monnina Obtusifolia fruit were identified for the first time: one pyroanthocyanin and three anthocyanins derived from delphinidin, cyanidin and peonidin. The relative contribution of different types of fractions isolated from the fruit (raw extracts, purified and pure compounds) on their antioxidant properties was also evaluated. The anthocyanin with greater power to capture the synthetic radical ABTS ${ }^{+}$was the compound derived from delphinidin. It was found that aglycones with only one $\mathrm{OH}$ group in the $\mathrm{B}$ ring $\left(4^{\prime}-\mathrm{OH}\right)$ have lower antioxidant activity; compared to compounds possessing 3 ', 4' di-OH substituted group such as delphinidin and cyanidin derivatives. The analysis of the reducing capacity of Folin-Ciocalteau and the antioxidant capacity equivalent to trolox of the isolated fractions suggest that the fruit of Monnina Obtusifolia can be considered as an important source of bioactive compounds, for its use in the industry.
\end{abstract}

Keywords: Monnina obtusifolia; anthocyanins; antioxidants; mass spectrometry; nuclear magnetic resonance 


\section{INTRODUCCIÓN}

El nombre científico del Ivilan ó Iguillán es Monnina obtusifolia H.B.K; pertenece a la familia Polygalaceae y al género Monnina el cual consta de unas 200 especies distribuidas desde el sureste de los Estados Unidos hasta Argentina, en la zona andina se encuentran unas 32 especies, sobre los 2400 m.s.n.m se han registrado 24 especies arbustivas, entre ellas, M. obtusifolia H.B.K. En Colombia esta especie está distribuida a lo largo de la región andina, a una altura entre los 2500 y 3300 m.s.n.m, en la zona del departamento de NariñoColombia, se encuentra en los municipios de Ipiales, Túquerres, Cumbal, Guachucal, Genoy y Nariño. La planta se describe como un arbusto de crecimiento lento que alcanza una altura entre 1 y 2 metros, con hojas alternas pecioladas y sus flores son de aspecto papilionáceo de color morado o rojizo. Su fruto en estado maduro es de color azul purpura, contiene la semilla embebida en una pulpa del mismo color y tiene una apariencia muy semejante al mortiño (Vaccinium meridionale). La forma varía entre redonda y ovalada y pesa alrededor de 0.100-0.180 g, los pobladores de la zona lo consumen ya que tiene un sabor agradable. Tradicionalmente su fruto se usa para el tratamiento de herpes simple y la curación de la Olanda (llagas en la boca). Por su matiz atractivo, brillante y por su solubilidad en agua, este fruto puede ser una fuente potencial de antocianinas que eventualmente puede remplazar a los colorantes sintéticos usados en alimentos.

Las antocianinas son pigmentos solubles en agua que pertenecen a la familia de los flavonoides (compuestos fenólicos), son glicosidos de antocianidinas derivados del 2-fenilbenzopirilio (ion flavilio) (Welch et al., 2008); que contienen dos anillos aromáticos ( $\mathrm{A}$ y $\mathrm{B}$ ) separados por un oxígeno que está formando un anillo heterocíclico de seis miembros (anillo C) (Zhao et al., 2017). Dentro de este grupo de compuestos también se han identificado piroantocianinas; moléculas que tienen un cuarto anillo heterocíclico entre los carbonos 4 y 5 (Mateus et al., 2003). Debido a la carga positiva del átomo de oxígeno en su molécula, las antocianinas tienen propiedades antioxidantes como donadores de hidrógeno (Kong et al., 2003). Generalmente las antocianinas están esterificadas con carbohidratos (azúcares) en la posición 3; los azúcares más comunes enlazados a antocianidinas son glucosa, galactosa, arabinosa, ramnosa y xilosa, aunque también pueden contener oligosacáridos como rutinosa, gentobiosa y soforosa; además, a estos azúcares podrían enlazarse diferentes ácidos orgánicos; los más comunes son ferúlico, cumárico, cinámico y malónico.

Las antocianinas son comúnmente sintetizadas por las plantas y son las responsables, bajo diferentes condiciones de $\mathrm{pH}$, de los colores rojo, naranja, azul y púrpura de muchas flores, frutas y verduras (Bueno et al., 2012; Flores et al., 2016; Flores., 2017). La presencia de antocianinas en diversas partes de la planta es de gran importancia, se sabe que las antocianinas juegan un papel importante en la ecofisiologia y en la propagación de plantas; ayudan a atraer polinizadores y participan en los mecanismos de defensa de las plantas contra factores de estrés bióticos y ambientales (Menzies et al., 2016). A las antocianinas y sus derivados se les han atribuido diferentes beneficios para la salud humana (He et al., 2010), se ha encontrado que son antioxidantes (Wang et al., 2018); anticancerígenos (Bontempo et al., 2015); tienen actividad antidiabética (Hong et al., 2013); contra la obesidad (Xie et al., 2018); previenen enfermedades cardiovasculares (Cassidy, 2018) y tienen potencial para prevenir la pérdida de memoria y enfermedades neurodegenerativas (Gutierres et al., 2014). Bajo esta perspectiva, es de gran importancia buscar nuevas fuentes de este tipo de moléculas bioactivas. Este estudio centra su interés en el estudio de los pigmentos del fruto de Ivilan, que eventualmente podrían ser aprovechados como fuente de compuestos bioactivos para su uso en la industria farmacéutica o de alimentos.

Estudios sobre esta planta han demostrado que el extracto metanólico de las hojas de Monnina obtusifolia H.B.K tiene actividad anti-angiogénica, antibacterial, anti cáncer e inhibe el crecimiento placental. Se ha encontrado además que las hojas contienen saponinas, xantonas, esteres de oligosacáridos y flavonoides (Lepore et al., 2011). Sin embargo, no existen investigaciones sobre los componentes que generan el color de la fruta, por lo que es de gran interés estudiar las características estructurales y capacidad antiradicalaria de este tipo de moléculas. Así, con el objetivo de valorar los polifenoles del fruto como fuente de compuestos bioactivos, en este trabajo de investigación el fruto de Monnina obtusifolia H.B.K se sometió a una caracterización química., para ello inicialmente se realizó un fraccionamiento y purificación de las antocianinas mayoritarias por UV-Vis, HPLC-ESI-MS y ${ }^{1} \mathrm{H}$-RMN y posteriormente el estudio se complementó con la determinación de la capacidad antioxidante contra el radical ABTS + de cada fracción y compuesto purificado.

\section{MATERIALES Y MÉTODOS}

Los frutos se cosecharon en la vereda Ipualpud del municipio de Guachucal - Departamento de Nariño-Colombia. Las características del arbusto y la clasificación taxonómica se evaluaron en el Herbario de la Universidad de Nariño. Se colectó una muestra representativa de la población vegetal, colectando frutos en su estado de maduración fisiológico, de igual consistencia, tamaño y características visuales. Mediante un modelo de muestreo aleatorio simple de 10 plantas, se recolectó un peso aproximado de $2 \mathrm{Kg}$. Los frutos se lavaron con agua, se homogenizaron y seleccionaron para el estudio $1000 \mathrm{~g}$, posteriormente se refrigeraron a $4^{\circ} \mathrm{C} \pm 1$ hasta su análisis. 


\section{Reactivos}

Los reactivos Folin-Cicalteau, acido gálico, Trolox, metanol, $\mathrm{HCl}$, acetona, acetonitrilo grado HPLC, agua grado HPLC, Trolox, ABTS se adquirieron de Sigma-Aldrich. La fase estacionaria Sephadex LH-20 se adquirió de Sigma Aldrich.

\section{Fraccionamiento cromatográfico de los polifenoles}

A partir de los frutos seleccionados, inicialmente se aisló un extracto metanólico que se denominó extracto crudo de Ivilan (EIC), luego con el propósito de aislar una muestra enriquecida en polifenoles, el EIC se sometió a un proceso de purificación mediante retención selectiva sobre columna cromatográfica, logrando un extracto de Ivilan purificado (EIP), posteriormente a partir del EIP se aisló., mediante cromatografía de exclusión por tamaño, una muestra rica en antocianinas monoméricas (ERM) y una fracción polimérica. Finalmente a partir del ERM se purificaron las antocianinas mayoritarias del fruto de Ivilan. A continuación se presentan los detalles metodológicos de este proceso:

Para el obtener el extracto crudo de Ivilan (EIC), inicialmente los frutos frescos y maduros $(1 \mathrm{~kg})$ se lavaron y posteriormente los frutos se maceraron por 8 horas en metanol-ácido acético (19:1, v/v); luego se centrifugó durante $10 \mathrm{~min}$ a $10.000 \mathrm{rpm}$ y se filtró en un embudo Buchner; usando como medio filtrante papel con poro $11 \mu \mathrm{m}$. El solvente se removió al vacío a $40{ }^{\circ} \mathrm{C}$ y el residuo se liofilizó; este extracto se diluyó en agua (1:1 $\mathrm{w} / \mathrm{w}$ ) y porciones separadas de $100 \mathrm{~g}$ (disueltas en $100 \mathrm{ml}$ de agua) se cargaron sobre una columna abierta que contenía como fase estacionaria un absorbente polimérico no iónico (Amberlita XAD-7) de dimensiones $80 \times 4 \mathrm{~cm}$ d.i. Los pigmentos adsorbidos se lavaron con agua destilada y se eluyeron con $1 \mathrm{~L}$ de metanolácido acético (19:1 v/v); de acuerdo con el procedimiento descrito por Degenhardt et al. (2000), el eluente se concentró al vacío a $40^{\circ} \mathrm{C}$ y la fase acuosa se liofilizó. El producto final fue un extracto de Ivilan purificado enriquecido en polifenoles (EIP). Por último, los taninos y las antocianinas del EIP se aislaron mediante el procedimiento descrito por Henke (1996).La muestra se disolvió en metanol; a continuación esta solución fue sometida a cromatografía de exclusión por tamaño utilizando una columna empacada con Sephadex LH-20 Sigma $(40 \times 3 \mathrm{~cm})$. La fracción de compuestos de bajo peso molecular (extracto de Ivilan rico en antocianinas monoméricas, ERM) se eluyó con una mezcla de metanol-agua (1:1) que contenía ácido trifluoroacético (TFA) al $0.1 \%$ y la fracción polimérica fue eluída con una mezcla de acetona-agua (2:1); con TFA al 0.1\%. La fracción monomérica ERM se analizó por cromatografía líquida de alta eficiencia acoplada con espectrometría de masas HPLC-DAD-ESI-MS. Posteriormente las antocianinas mayoritarias se purificaron mediante HPLC semi-preparativa y se analizaron por ${ }^{1} \mathrm{H}-\mathrm{RMN}$.

\section{Caracterización de las antocianinas monoméricas por HPLC-ESI-MS}

La identificación parcial de los componentes se realizó en un espectrómetro de masas LCMS Shimadzu QP 2010; equipado con una interface electroespray. El voltaje del detector fue de $1.5 \mathrm{KV}$, flujo del gas nebulizador de $4.5 \mathrm{~L} / \mathrm{min}$, columna Phenomenex Luna $5 \mu \mathrm{m} \mathrm{C18} \mathrm{100A,} 250 \times 4.6 \mathrm{~mm}$, longitud de onda en detector UV $520 \mathrm{~nm}$. Fase móvil A: Acetonitrilo:ácido fórmico:agua 3:10:87 (v/v/v); B: acetonitrilo:ácido fórmico:agua 50:10:40 (v/v/v). Gradiente: 6-20 \%B; 0-20 min, 20-40 \%B; 20-35 min, 40-60 \%B; 35-40 min, 60-90\%B; $40-$ $45 \mathrm{~min}, 90-6 \% \mathrm{~B}$; 45- $60 \mathrm{~min}$. Flujo: $0.8 \mathrm{~mL} / \mathrm{min}$. Las muestras se disolvieron en $50 \mu \mathrm{L}$ de la fase móvil B. Volumen de inyección de $5 \mu \mathrm{L}$. La cromatografía HPLC en modo semi-preparativo se realizó utilizando una columna Luna $\mathrm{C}_{18}(10 \mathrm{~mm} \times 250 \mathrm{~mm}$ : $5 \mu \mathrm{m}$ espesor de la película) y un detector UV 6000LP. Se aplicó un perfil de elución isocrático $(95 \% \mathrm{~A}, 5 \% \mathrm{~B})$, usando acetonitrilo, ácido fórmico y agua (Solvente A: 3:10:87, $\mathrm{v} / \mathrm{v} / \mathrm{v}$; solvente B: 50:10:40, v/v/v), la velocidad de flujo fue de $4 \mathrm{ml} / \mathrm{min}$ por $20 \mathrm{~min}$, inyectando alícuotas de 40 $\mu \mathrm{L}(250 \mathrm{mg} / \mathrm{ml})$.

\section{Resonancia magnética nuclear de las antocianinas}

Las antocianinas aisladas se disolvieron en una mezcla de metanol-d4-TFA-d1 (19:1, v/v) y los espectros de ${ }^{1} \mathrm{H}-\mathrm{RMN}$ se midieron en un equipo Bruker AMX-500.

\section{Cuantificación de antocianinas monoméricas}

El contenido de antocianinas monoméricas se calculó por cuantificación fotométrica; utilizando un espectrofotómetro (Merck, Spectroquant ${ }^{\circledR}$ Pharo 300, USA). El análisis se realizó usando el método del pH diferencial (Giusti y Wrolstad, 2003) y la concentración del pigmento se expresó como mg de cianidina 3glucósido/g de extracto; usando la ecuación (1). 


$$
(\mathrm{mg} / \mathrm{g})=(\mathrm{A} \times \mathrm{FD} \times \mathrm{PM} \times \mathrm{V}) /(\varepsilon \times \mathrm{L})
$$

Donde A: absorbancia a $520 \mathrm{~nm}, \mathrm{FD}$ : factor de dilución, PM: peso molecular (cianidina 3-glucósido $=449.2$ $\mathrm{g} / \mathrm{mol}$ ), V: volumen del extracto, $\varepsilon$ : coeficiente de extinción molar (cianidina 3-glucósido $=26900 \mathrm{~L} / \mathrm{mol} . \mathrm{cm}$ ) y $L$ : longitud de la celda $(1 \mathrm{~cm})$.

\section{Capacidad reductora de Folin-Ciocalteau (CRFC)}

La capacidad de reducción de Folin-Ciocalteau de los diferentes extractos de Ivilan (Monnina Obtusifolia) se evaluó por el método de Folin-Ciocalteau (Singleton et al., 1999, Hurtado y Perez., 2014). Se mezcló una alícuota de $100 \mu \mathrm{L}$ del extracto metanólico con $900 \mu \mathrm{L}$ del reactivo de Folin-Ciocalteau y la solución se mantuvo por 5 min a temperatura ambiente. Posteriormente, luego de la adición de $750 \mu \mathrm{L}$ de bicarbonato de sodio; la solución se agitó por 30 segundos y se mantuvo en reposo por 90 min a temperatura ambiente. Finalmente, se midió la absorbancia a $765 \mathrm{~nm}$ utilizando un espectrofotómetro (Merck, Spectroquant ${ }^{\circledR}$ Pharo 300 , USA) y los resultados se expresaron en miligramos de equivalentes de ácido gálico por litro (mg EAG/L). Posteriormente la capacidad de reducción de Folin-Ciocalteau se expresó como miligramos de ácido gálico por 100 gramos de extracto seco.

\section{Determinación de la capacidad antioxidante equivalente al Trolox (TEAC)}

La capacidad antioxidante se midió in Vitro con base en la habilidad de la muestra para capturar el radical

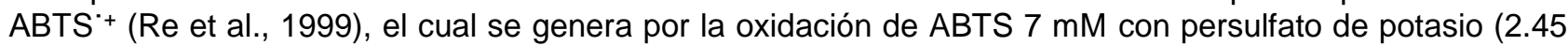
$\mathrm{mM}$ ) en agua. La solución de ABTS + se diluyó con boffer-fosfato (SBF) a pH 7.4 y luego fue almacenada por 16 horas en la oscuridad y a temperatura ambiente; hasta alcanzar una absorbancia de $0.70 \pm 0.02$ at 734 $\mathrm{nm}$. Luego, $30 \mu \mathrm{L}$ de cada muestra se mezcló con $3 \mathrm{ml}$ de solución diluida de ABTS'+ y se agitó por 1 min. Después de esperar $6 \mathrm{~min}$, se midió la absorbancia a $734 \mathrm{~nm}$ en un espectrofotómetro UV-Vis (Merck, Spectroquant ${ }^{\circledR}$ Pharo 300 , USA). Los datos se obtuvieron por interpolación de la absorbancia en la curva de calibración de Trolox (0.5-3.0 mM) y los resultados se expresaron como mmol de equivalentes Trolox/g de extracto seco.

\section{Análisis estadístico}

Todos los análisis se realizaron por triplicado. El análisis estadístico se realizó usando Statgraphics Centurion 16.1.15, para el análisis unifactorial de varianza se usó el procedimiento ANOVA. Las diferencias significativas $(P<0.05)$ entre las medias se determinaron usando la prueba de múltiples rangos.

\section{RESULTADOS Y DISCUSIÓN}

El fraccionamiento inicial del fruto permitió establecer que la cantidad de muestra soluble en metanol-ácido acético (19:1) (extracto de Ivilan crudo, EIC) corresponde al $25.1 \%$ del fruto y la fracción retenida sobre la resina XAD-7 representa el $6.5 \%$ del fruto (extracto de Ivilan purificado rico en polifenoles, EIP). En la tabla 1 se muestra la CRFC y el contenido de antocianinas monoméricas para estos dos extractos (EIC y EIP). Mediante el análisis ANOVA se observó que en estas dos fracciones existen diferencias significativas en la CRFC ( $p<0.05)$, siendo la CRFC del EIP (18.46 mg EAG/g muestra) significativamente mayor que la del EIC, lo que demuestra la eficiencia de la columna para retener selectivamente los polifenoles de la muestra. Igual comportamiento se observa para el contenido de antocianinas de los extractos (Tabla 1).

Tabla 1: Capacidad reductora de Folin-Ciocalteau (CRFC), contenido de antocianinas y actividad antioxidante TEAC de las fracciones aisladas del fruto de Ivilan (Monnina Obtusifolia).

\begin{tabular}{|l|l|l|l|l|l|}
\hline \multicolumn{1}{|c|}{ Muestra } & A440/Avis & Aacil/Amax & \multicolumn{1}{c|}{$\begin{array}{r}\text { TEAC } \\
\text { (mmol trolox/g muestra) }\end{array}$} & $\begin{array}{c}\text { Antocianinas } \\
\text { (mg Cy-3-glu/g } \\
\text { muestra) }\end{array}$ & $\begin{array}{c}\text { CRFC } \\
\text { (mg EAG/g } \\
\text { muestra) }\end{array}$ \\
\hline EIC & - & - & $8.500 \pm 0.08$ & $3.81 \pm 0.02$ & $6.21 \pm 0.04$ \\
\hline EIP & - & - & $3.307 \pm 0.06$ & $18.03 \pm 0.09$ & $18.46 \pm 0.15$ \\
\hline Antocianina 1 & 33.3 & 68.3 & $0.691 \pm 0.04$ & - & - \\
\hline Antocianina 2 & 22.4 & 66.5 & $1.638 \pm 0.07$ & - & - \\
\hline Antocianina 3 & 32.8 & 67.4 & $1.039 \pm 0.05$ & - & - \\
\hline Antocianina 4 & 32.5 & 61.3 & $1.136 \pm 0.06$ & - & - \\
\hline Ácido ascórbico & - & - & $\begin{array}{l}0.970 \pm 0.01(\mathrm{mmol} \text { trolox/mol) } \\
5.5 \times 10^{-3} \mathrm{mmol} \text { trolox/g }\end{array}$ & - & - \\
\hline
\end{tabular}


Mediante el método del pH diferencial se encontró que el contenido de antocianinas monoméricas en el fruto es de $95.3 \pm 0.6 \mathrm{mg} \mathrm{Cy}-3-\mathrm{glu} / 100 \mathrm{~g}$ de fruto fresco y la capacidad reductora de Folin-Ciocalteau (CRFC) expresada en $\mathrm{mg}$ EAG/100 $\mathrm{g}$ de fruto es de $155.3 \pm 6.2$ que es mayor a la CRFC reportada para la fresa (132.1 mg EAG /100 g de fruta), uva (117.1 mg EAG/100 g de fruta) y guayaba (83 mg EAG/100 g de fruta), pero inferior a frutos como la Zarzamora: $248.5 \mathrm{mg}$ EAG $/ 100 \mathrm{~g}$ de fruta y mortiño: $281.6 \mathrm{mg}$ EAG/100 g de fruta (Kuskoski et al., 2004). Estos datos confirman el potencial uso de este fruto como fuente de colorantes naturales.

\section{Caracterización de las antocianinas monoméricas por HPLC-ESI-MS}

La cromatografía en fase reversa con gradiente de elución ha sido ampliamente utilizada para la separación y caracterización de compuestos fenólicos. Las condiciones cromatográficas usadas en este trabajo permitieron una buena separación de las antocianinas de la muestra y la identificación parcial de los pigmentos se confirmó por el ion molecular generado en espectrometría de masas con ionización electrospray en modo positivo (HPLC-ESI-MS).

Mediante el proceso de fraccionamiento adoptado, se aislaron las antocianinas monoméricas del EIP. Los compuestos polifenólicos del EIP se sometieron a fraccionamiento diferencial basado en su hidrofobicidad. De esta forma los compuestos menos hidrofóbicos; fracción menos retenida en Sephadex (antocianinas monoméricas), eluyeron de la columna en presencia de solventes más polares; metanol-agua (1:1) que contenía TFA al $0.1 \%$. La fracción más retenida en Sephadex (fracción polimérica) se eluyeron con acetonaagua (2:1) que contenía ácido TFA al $0.1 \%$. En la figura 1 se muestra el cromatograma HPLC-ESI-MS de la fracción rica en antocianinas monoméricas aislada del fruto de Ivilan (Monnina Obtusifolia), se observan cuatro antocianinas mayoritarias que representan $\sim 97.6 \%$ del área total, el compuesto 1 corresponde al 13.7\%, el compuesto 2 el $60.9 \%$ (compuesto mayoritario), el 3 (13.7\%) y el 4 el $9.3 \%$.

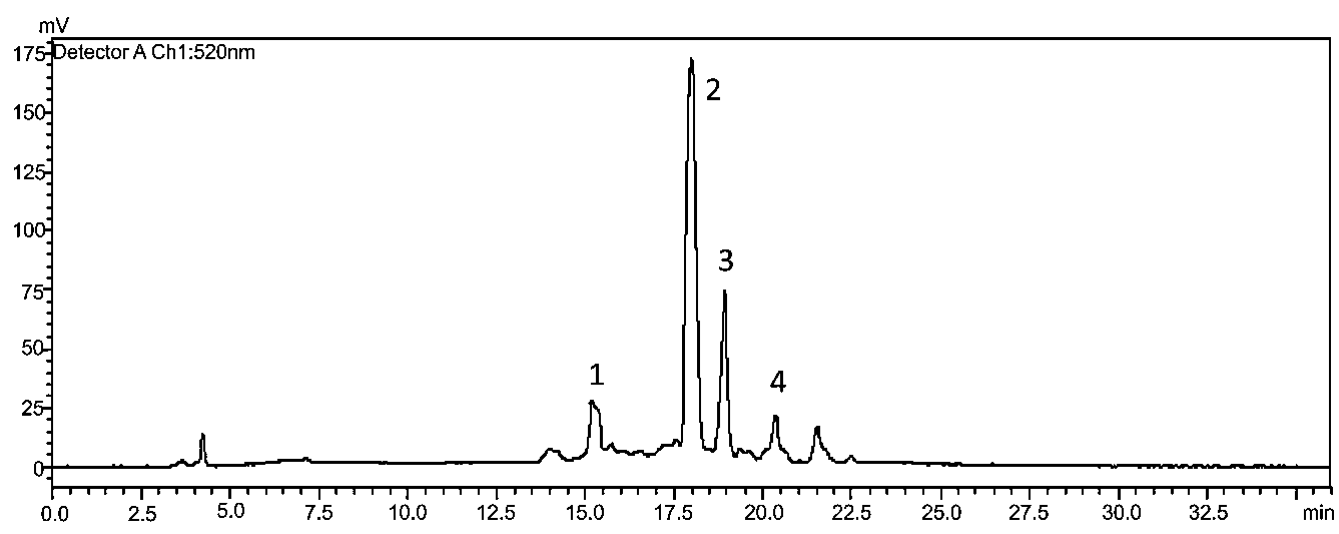

Fig. 1: Cromatograma HPLC-ESI-MS de la fracción rica en antocianinas monoméricas aislada del fruto de Ivilan (Monnina Obtusifolia).

El extracto eluído de la columna de Sephadex (ERM) posteriormente se sometió a HPLC semi-preparativa, lográndose purificar los compuestos 1-4 (Figura1). Finalmente los compuestos purificados de caracterizaron por UV-Vis, HPLC-ESI-MS y ${ }^{1} \mathrm{H}-\mathrm{RMN}$. A partir de los espectros UV-Vis se logró elucidar alguna información estructural de dichos compuestos, se ha publicado que la longitud de onda de máxima absorción de las antocianinas se encuentra entre 500 y $530 \mathrm{~nm}$ (Giusti et al., 1999). Además, se ha encontrado que en la región visible; los derivados acilados de este tipo de pigmentos no muestran diferencia con respecto a los correspondientes no acilados, sin embargo; en la región del ultravioleta suelen presentar un máximo pronunciado en el intervalo $310-335 \mathrm{~nm}$, correspondiente a la absorción del grupo acilo (Fossen y Andersen, 1999).

El análisis por UV-Vis del compuesto 1 presenta un máximo de absorción $(\lambda \max )$ en $527 \mathrm{~nm}$. El máximo de absorción localizado en la region de 500-530 nm y el cambio de color observado al variar el pH de una solución acuosa de este pigmento índican la presencia de pigmentos tipo antocianina. La absorción en la región 310$335 \mathrm{~nm}$ permite afirmar que hay acilación en esta molécula. De otro lado, para este pigmento se encontró una relación $A_{440} / A_{\text {vis-max }}$ de $33.3 \%$ y una $A_{\text {aciil }} / A_{\max }$ de $68.3 \%$ que evidencia una glicosidación en la posición $\mathrm{C}_{3}$ de esta molécula. La literatura reporta además que una relación $A_{440} / A_{v i s}$ entre 20-35\%, sugiere una glicosidación en la posición 3 de la aglicona (Giusty et al., 1999) y una relación $A_{a c i l} / A_{\max }$ con valores entre 50$70 \%$ evidencia la presencia de grupos acilo (Wrostad et al., 2000); por lo tanto, los resultados muestran que todos los compuestos (1-4) presentan no solo sustitución en la posición 3 sino que también poseen grupos acilo (Tabla 1). 
De acuerdo con los resultados del análisis HPLC-ESI-MS, la antocianina 1 con tR 15.2 min y $\lambda \max 538 \mathrm{~nm}$ se identificó por su peso molecular $\left(\mathrm{M}^{+}\right)$y sus fragmentos en el espectro de masas. El espectro de este pigmento mostró un $\mathrm{M}^{+}$en $\mathrm{m} / \mathrm{z} 775 \mathrm{u}\left(\mathrm{C}_{36} \mathrm{H}_{39} \mathrm{O}_{19}\right)$ e iones fragmento en $\mathrm{m} / \mathrm{z} 529$ y $325 \mathrm{u}$. El ion fragmento en $\mathrm{m} / \mathrm{z} 529 \mathrm{u}$ puede explicarse por la pérdida de una hexosa (azúcar I) y un grupo acilo (ácido succínico) ([M-146-100]+) y el ion fragmento en $\mathrm{m} / \mathrm{z} 325 \mathrm{u}$ permite postular que este compuesto tiene como aglicona a la vinilpiranopeonidina. El ion correspondiente a la aglicona se genera por la pérdida adicional de un grupo acetilo (43u) unido a una hexosa (162u, azúcar II) ([M+H-146-100-43-162]+).

Para la antocianina 1 el espectro RMN-1 $\mathrm{H}$ (Bruker AMX-500 MHz (metanol-d4-TFA-d 1 (19:1, v/v) mostró los desplazamientos químicos en ppm: $6.66(1 \mathrm{H}, \mathrm{s}, \mathrm{H}-6), 6.80(1 \mathrm{H}, \mathrm{s}, \mathrm{H}-8), 8.01\left(1 \mathrm{H}, \mathrm{s}, \mathrm{H}-2^{\prime}\right), 8.89\left(1 \mathrm{H}, \mathrm{s}, \mathrm{H}_{-} 5^{\prime}\right)$, $8.26\left(1 \mathrm{H}, \mathrm{s}, \mathrm{H}-6^{\prime}\right), 3.75\left(3 \mathrm{H}, \mathrm{OCH}_{3}\right), 7.00(1 \mathrm{H}, \mathrm{sa}, \mathrm{H}-11), 8.40(1 \mathrm{H}, \mathrm{d}, \mathrm{H}-12), 5.27\left(1 \mathrm{H}, \mathrm{d}, \mathrm{J}=8.73 \mathrm{~Hz} \mathrm{H}-1^{\prime \prime}\right)$, $4.18\left(1 \mathrm{H}, \mathrm{m}, \mathrm{H}-2\right.$ "), $3.58\left(1 \mathrm{H}, \mathrm{m}, \mathrm{H}-3\right.$ "), $3.41\left(1 \mathrm{H}, \mathrm{m}, \mathrm{H}-4\right.$ "), $3.83\left(1 \mathrm{H}, \mathrm{m}, \mathrm{H}-5\right.$ "), $5.02\left(\mathrm{H}, \mathrm{d}, \mathrm{J}=3.85 \mathrm{~Hz} \mathrm{H}-1^{\prime \prime \prime)}\right.$ ), $3.91(\mathrm{H}, \mathrm{m}, \mathrm{H}-2$ '”), 3.68 (H, m, H-3"'), $3.34(\mathrm{H}, \mathrm{m}, \mathrm{H}-4$ '”), $1.13(\mathrm{H}, \mathrm{d}, \mathrm{H}-6$ '”). Grupo: acetil: 2.32 (1H, s, H-2), grupo succinil: $2.53(1 \mathrm{H}, \mathrm{s}, \mathrm{H}-3)$. Estos datos permitieron confirmar algunas características estructurales de las moléculas, a campo bajo del espectro de ${ }^{1} \mathrm{H}$-RMN se observaron cinco señales $(6.66,6.80,8.01,8.89$ y $8.26 \mathrm{ppm}$ ). Con base en el desplazamiento químico y patrones de acoplamiento, la señal a 6.66 y $6.80 \mathrm{ppm}$ se asignó a H-6 y H-8 respectivamente (Figura 2).
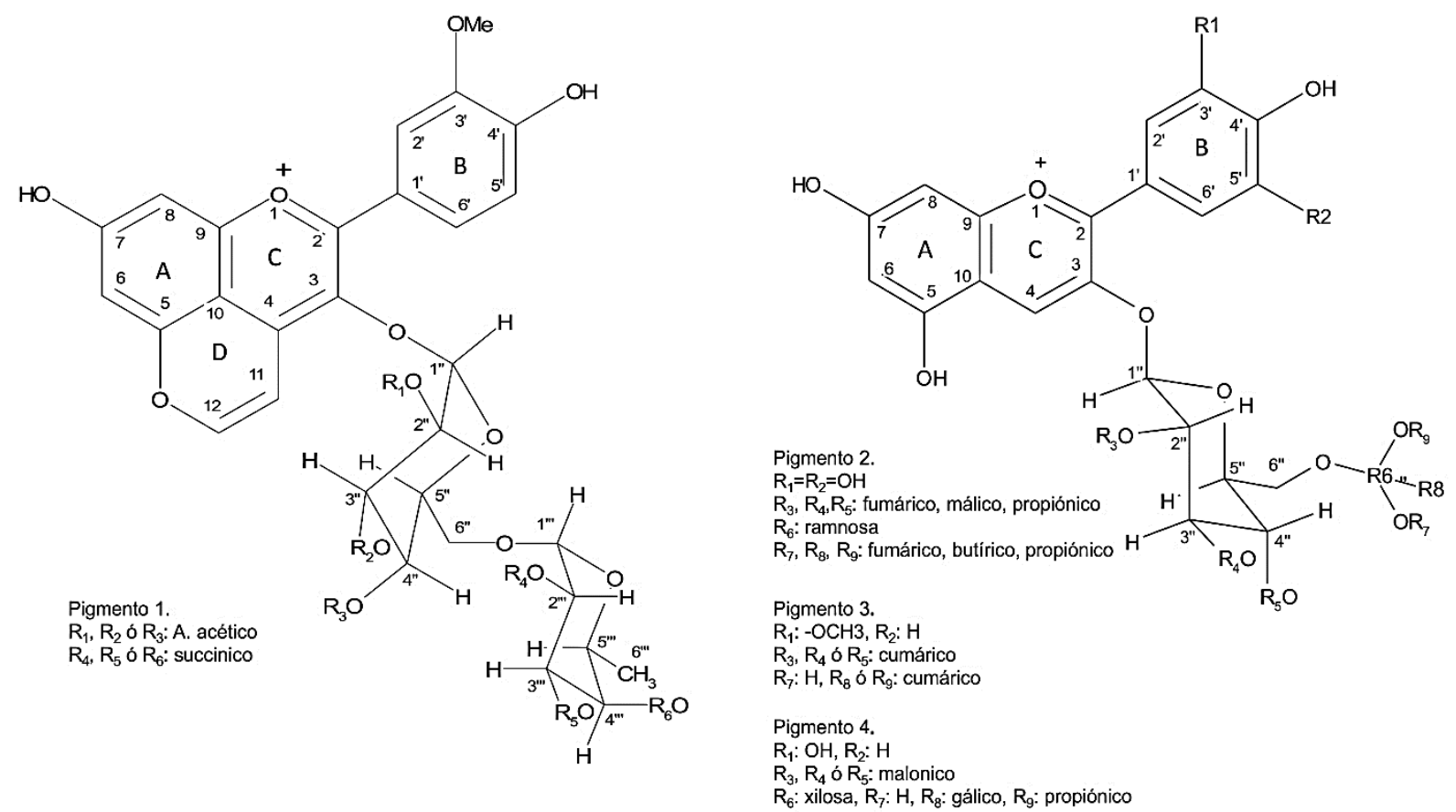

Fig. 2: Antocianinas mayoritarias identificadas en Monnina Obtusifolia.

Además, los 3H a 8.01, 8.89 y 8.26 se asignaron individualmente como H-2', H-5' y H-6' y el singlete a campo alto en $3.75 \mathrm{ppm}$ corresponde a los protones del grupo $-\mathrm{OCH}_{3}$. La ausencia de señal para $\mathrm{H}-4$ en la aglicona propuesta y la presencia de señales en $\mathrm{H}-11$ (singlete ancho en $7.00 \mathrm{ppm}$ ) y H-12 (doblete en $8.40 \mathrm{ppm}$ ) de la estructura pirano confirman la identidad de la aglicona como vinilpiranopeonidina. También en la zona de 3 a 5.5 ppm del espectro se observan señales características a las subunidades de azúcar. En esta zona se evidencia la presencia de dos unidades de azúcar por las señales en $\delta H 5.27$ (d, J=8.73); 5.02 (d, J=3.85) correspondientes a protones anoméricos, comprobando así la hipótesis planteada en el análisis por ESI-MS (dos subunidades de azúcar).

La configuración de los carbonos anoméricos fue establecida a través de la comparación de las constantes

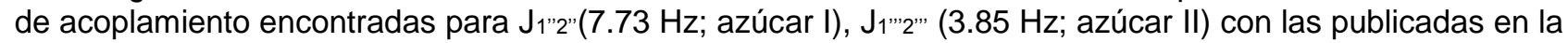
literatura: configuración $\alpha 1.5 \mathrm{~Hz}$ y configuración $\beta 7.7 \mathrm{~Hz}$ (Andersen y Fossen, 2003); configuración $\alpha 1-4 \mathrm{~Hz}$, configuración $\beta$ 6-8 Hz (Agrawal, 1992); configuración $\alpha 3 \mathrm{~Hz}$, configuración $\beta 7.5 \mathrm{~Hz}$ (Matsufuji et al., 2003). De esta forma se estableció la configuración $\beta$ del azúcar I (unida al $\mathrm{C}_{3}$, figura 2)) y $\alpha$ de la otra unidad de azúcar. La señal en 1.23 (d, H-6"') correspondiente a un grupo metilo permite postular que la segunda unidad de azúcar posee este grupo en su estructura, lo que permite postular la presencia de ramnosa. Las demás señales son características de los azúcares I y II; permitiendo establecer que estas subunidades pueden ser glucosa y ramnosa respectivamente; desplazamientos que están de acuerdo con las publicadas en la literatura (Cabrita y Andersen, 2000). 
Con base en las deducciones anteriores el pigmento 1 se identificó tentativamente como una piranoantocianidina: vinilpiranopeonidin-3-O- $\beta$-(acetil) glucopiranosil-O- $\alpha-($ succinil) ramnosido. Las piranoantocianinas pertenecen a un grupo importante de pigmentos derivados de antocianinas que están presentes principalmente en frutas, alimentos procesados o en vino rojo (Rentzsch et al., 2007). Debido a su nuevo anillo pirano, se ha encontrado que este tipo de moléculas son mucho más estables hacia las variaciones de $\mathrm{pH}$ y blanqueamiento con $\mathrm{SO}_{2}$. (Mateus et al., 2003).
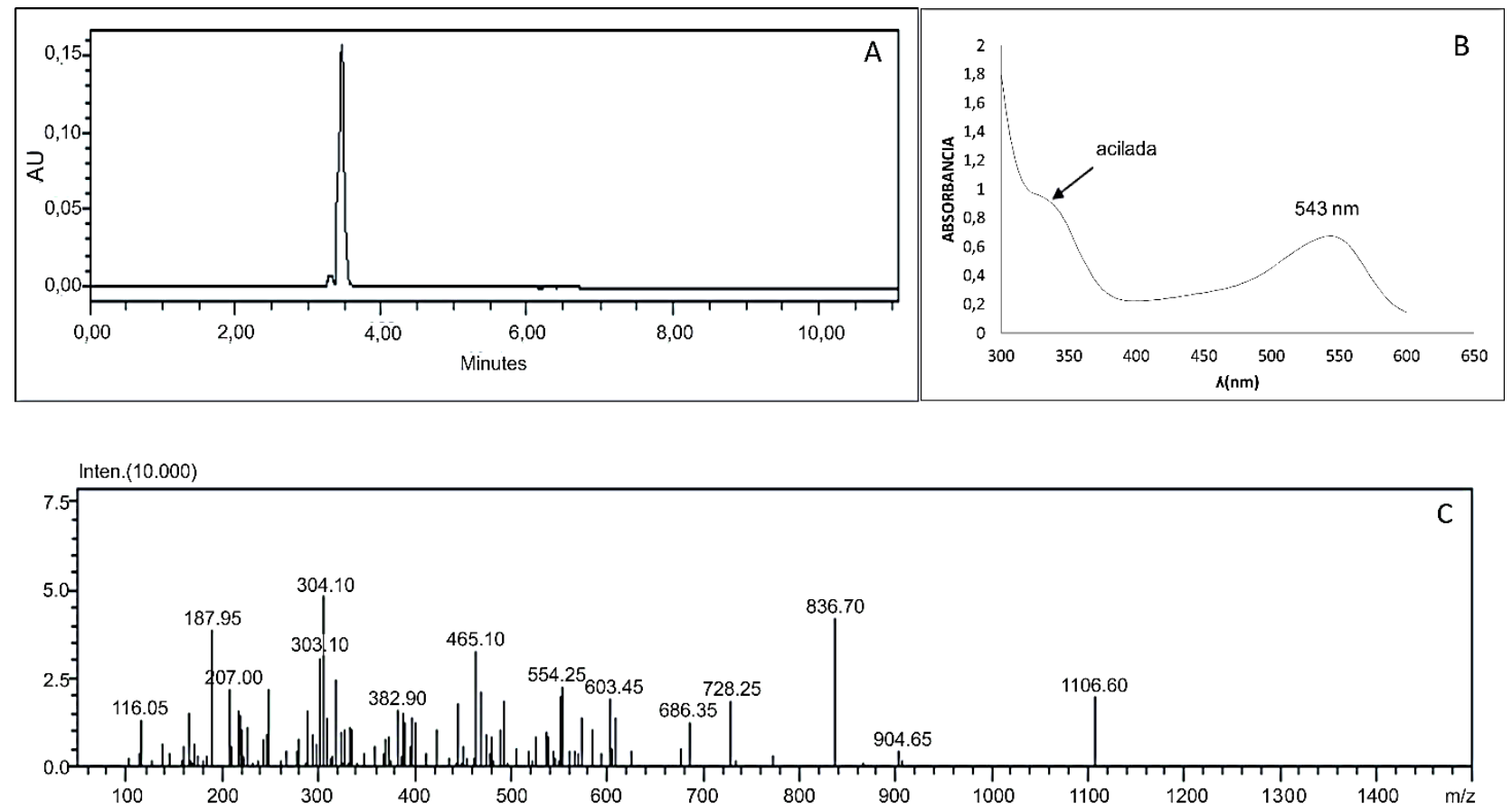

Fig.3: (A) Cromatograma HPLC-DAD $(520 \mathrm{~nm})$ de la antocianina 2 aislada y purificada a partir de Monnina Obtusifolia, (B) Espectro UV-Vis, (C) Espectro de masas HPLC-ESI-MS.

Para la antocianina 2 (compuesto mayoritario) con th de 18.0 min y $\Lambda_{\max } 543 \mathrm{~nm}$; el espectro de masas mostró un ion molecular $\mathrm{M}^{+}$en $\mathrm{m} / \mathrm{z} 1106 \mathrm{u}\left(\mathrm{C}_{49} \mathrm{H}_{54} \mathrm{O}_{29}\right)$ y iones fragmento en $\mathrm{m} / \mathrm{z} 904,836,728,465$ y $303 \mathrm{u}$. El ion fragmento en $\mathrm{m} / \mathrm{z} 303 \mathrm{u}$ sugiere para este compuesto la presencia de delfinidina como aglicona. Este fragmento puede explicarse por la pérdida de $803 \mathrm{u}$ lo que sugiere la perdida de dos azúcares (hexosa y pentosa), dos unidades de ácido fumárico, ácido málico, dos unidades de ácido propiónico y ácido butírico [M-162-146- 2x98-116- 2x56- 71 $]^{+}$. El ion fragmento en $\mathrm{m} / \mathrm{z}$ 904u se origina posiblemente por la pérdida de una hexosa y una unidad de ácido propiónico [M-146-56]+. El ion en $\mathrm{m} / \mathrm{z} 836 \mathrm{u}$ se genera por la pérdida de los ácidos málico, fumárico y propiónico [M-116-98-56]+. El ion en $\mathrm{m} / \mathrm{z} 728$ sugiere la pérdida de $379 \mathrm{u}$ : dos unidades de ácido propiónico, dos unidades de ácido fumárico y una unidad de ácido butítrico [M- 2x56-2x98$71]^{+}$. El ion fragmento en $\mathrm{m} / \mathrm{z} 465 \mathrm{u}$ sugiere la presencia de la delfinidina (303u) unida a una hexosa (162u, posiblemente glucosa) (Figura 3).

Así mismo con base en el desplazamiento químico observado en el espectro de ${ }^{1} \mathrm{H}-\mathrm{RMN}$ para pigmento 2 , se comprobaron algunas características estructurales de la molécula. Los desplazamientos químicos para esta molécula fueron: $8.22(1 \mathrm{H}, \mathrm{s}, \mathrm{H}-4), 6.66(1 \mathrm{H}, \mathrm{s}, \mathrm{H}-6), 7.02(1 \mathrm{H}, \mathrm{s}, \mathrm{H}-8), 7.69(1 \mathrm{H}, \mathrm{s}, \mathrm{H}-2), 7.88(1 \mathrm{H}, \mathrm{s}, \mathrm{H}-$ 6'), $5.48\left(1 \mathrm{H}, \mathrm{d}, \mathrm{J}=7.87 \mathrm{~Hz}, \mathrm{H}-1\right.$ "), $3.57\left(1 \mathrm{H}, \mathrm{m}, \mathrm{H}-3\right.$ "), 3.59 (1H, sa, H-4"), $3.67\left(1 \mathrm{H}, \mathrm{m}, \mathrm{H}-5^{\prime \prime}\right), 3.78(1 \mathrm{H}, \mathrm{s}$, H-6"), $4.81(1 \mathrm{H}, \mathrm{d}, \mathrm{J}=7.39 \mathrm{~Hz}, \mathrm{H}-1$ '”), 4.38 (1H, d, H-2"'), $3.75(1 \mathrm{H}, \mathrm{d}, \mathrm{H}-4$ "'), $1.23(1 \mathrm{H}, \mathrm{d}, \mathrm{H}-6$ '”), grupo propionil: $0.8(1 \mathrm{H}, \mathrm{sa}, \mathrm{H}-2), 1.91(1 \mathrm{H}, \mathrm{d}, \mathrm{H}-3)$, fumarico $5.70(1 \mathrm{H}, \mathrm{d}, \mathrm{J}=12.1)$, málico $2.80(1 \mathrm{H}$, dd). Las señales a 7.69s y 7.88s ppm se asignaron a los protones de la aglicona $\mathrm{H}-2$ ' y $\mathrm{H}-6$ ' respectivamente, igualmente los $2 \mathrm{H}$ a 7.02 y $6.66 \mathrm{ppm}$ a H-8 y H-6, y un singlete en $8.22 \mathrm{ppm}$ a H-4 (Figura 2). Estos desplazamientos químicos confirman la delfinidina como aglicona, fue evidente también la presencia de 2 azúcares por las señales características de dos protones anoméricos, el primero aparece en la región de $5.48 \mathrm{~d}$ ppm $(\mathrm{J} 7.87 \mathrm{~Hz}$, valor característico de la configuración $\beta$ ) (azúcar 1); como se mencionó anteriormente según la relación $A_{440 / A_{\max }}$ este azúcar está unida a la posición 3 de la aglicona. El segundo protón anomérico aparece en la región de $4.81 \mathrm{ppm}$ como un doblete, con una constante de acoplamiento de $7.39 \mathrm{~Hz}$, valor característico de la configuración $\beta$ (azúcar 2), para este azúcar se observó además la presencia de un singlete correspondiente a un grupo metilo en la región de 1.23 ppm, que sugiere que este azúcar es la ramnosa. Los datos UV-Vis, ESI-MS y RMN-H permitieron identificar tentativamente a esta molécula como delfinidina-3-O- $\beta$-(fumaroil, malioil, propionil) glucopiranosil-O- $\beta$-(fumaroil, butiroil, propionil) ramnósido. 
El peso molecular de la antocianina $3\left(t_{R} 18.9 \min\right.$ y $\Lambda_{\max } 530 \mathrm{~nm}$ ) se estableció con base en la señal del ion molecular $\mathrm{M}^{+}$en $\mathrm{m} / \mathrm{z} 1037 \mathrm{u}\left(\mathrm{C}_{53} \mathrm{H}_{49} \mathrm{O}_{22}\right)$ y iones fragmento en $\mathrm{m} / \mathrm{z} 609 \mathrm{u}$ y 301 . El ion en $\mathrm{m} / \mathrm{z} 609 \mathrm{u}$ se origina por la pérdida de una hexosa y dos grupos acilo: p-cumárico y p-hidroxibenzoico [M-162-146-120]+. El ión en $\mathrm{m} / \mathrm{z} 301$ sugiere la presencia de una unidad de peonidina y se genera por la fragmentación del ion en $\mathrm{m} / \mathrm{z}$ 609u [609-162-146] ${ }^{+}$por la pérdida adicional de una hexosa y una unidad de ácido cumárico. Este análisis permitió postular a esta molécula como: Peonidin-3-O-(cumaroil) hexosa-O-(cumaroil, xidroxibenzoil) hexosa. Debido a la poca cantidad de muestra purificada por HPLC semi-preparativa, el compuesto 3 no se logró analizar por espectroscopia de Resonancia Magnética Nuclear protónica $\left({ }^{1} \mathrm{H}-\mathrm{RMN}\right)$.

Para la antocianina 4 con th 20.3 min y $\lambda \max 528 \mathrm{~nm}$ se encontró un peso molecular de $875 \mathrm{u}\left(\mathrm{C}_{39} \mathrm{H}_{39} \mathrm{O}_{23}\right)$ y iones fragmento en $\mathrm{m} / \mathrm{z} 535,449$ y $287 \mathrm{u}$, el ion fragmento en $\mathrm{m} / \mathrm{z} 535 \mathrm{u}$ corresponde a la pérdida de una pentosa (puede ser xilosa) y dos grupos acilo (ácido propiónico y gálico) [M-132-56-152] ${ }^{+}$. El ion en $\mathrm{m} / \mathrm{z} 449 \mathrm{u}$ $[\mathrm{M}-132-152-56-86 \mathrm{u}]^{+}$se origina posiblemente por la pérdida de ácido malónico y el fragmento en $\mathrm{m} / \mathrm{z} 287 \mathrm{u}$ establece que la aglicona de esta antocianina es la cianidina, este ion genera a partir del ion $\mathrm{m} / \mathrm{z} 449 \mathrm{u}$ por la pérdida adicional de una hexosa [M-132-56-152-86-162]+. Los desplazamientos químicos para esta molécula fueron: $8.33(1 \mathrm{H}, \mathrm{s}, \mathrm{H}-4), 6.68(1 \mathrm{H}, \mathrm{s}, \mathrm{H}-6), 6.95(1 \mathrm{H}, \mathrm{s}, \mathrm{H}-8), 7.55\left(1 \mathrm{H}, \mathrm{s}, \mathrm{H}-2^{\prime}\right), 7.09\left(1 \mathrm{H}, \mathrm{s}, \mathrm{H}-5^{\prime}\right), 8.26(1 \mathrm{H}$, s, H-6'), $5.48\left(1 \mathrm{H}, \mathrm{d}, \mathrm{J}=7.74, \mathrm{~Hz} \mathrm{H}-1^{\prime \prime}\right), 3.79\left(1 \mathrm{H}, \mathrm{sa}, \mathrm{H}-2^{\prime}\right), 3.54\left(1 \mathrm{H}, \mathrm{s}, \mathrm{H}-3\right.$ ") $, 3.53\left(1 \mathrm{H}, \mathrm{s}, \mathrm{H}-4{ }^{\prime \prime}\right), 3.84(1 \mathrm{H}$, sa, H-5"), 5.89 (1H, m, H-6"), 5.30 (1H, d, J = $7.71 \mathrm{~Hz}, \mathrm{H}-1$ '”), 3.31 (1H, sa, H-2"'), 3.56 (1H, sa, H-3"'), 3.80 (1H, sa, H-5"'), $1.19(1 \mathrm{H}, \mathrm{s}, \mathrm{H}-2), 1.91(1 \mathrm{H}, \mathrm{d}, \mathrm{H}-3)$. Grupo malonil: $3.42(1 \mathrm{H}, \mathrm{m}, \mathrm{H}-2)$, grupo galoil: $7.05(1 \mathrm{H}$, $\mathrm{s}, \mathrm{H}-2)$, grupo popionil: $0.8(1 \mathrm{H}, \mathrm{sa}, \mathrm{H}-2)$. Un análisis similar permitió identificar tentativamente a esta molécula como: Cianidina-3-O- $\beta$-(malonil) glucopiranosil-O- $\beta$-(galoil, propionil) xilosido.

\section{Capacidad antioxidante equivalente al Trolox (TEAC)}

La actividad de captura del catión radical $\mathrm{ABTS}^{+}+$ha sido extensamente usada para medir la capacidad antioxidante de diversos extractos de polifenoles, antocianinas y betalaínas. El radical ABTS ${ }^{+}$es reducido por los antioxidantes mediante la donación de hidrógeno y el cambio de color puede ser cuantificado por la disminución de la absorbancia a $\lambda=734 \mathrm{~nm}$. Finalmente, la habilidad relativa para reducir el ABTS ${ }^{+}$se compara con el estándar Trolox. En la tabla 1 se muestra la capacidad de las diferentes muestras (EIC, EIP y antocianinas purificadas) para reducir el catión radical ABTS ${ }^{+\cdot .}$. En los ensayos se usó como control positivo el ácido ascórbico, el valor TEAC publicado en la literatura para el ácido ascórbico es de $1.05 \mathrm{mmol}$ Trolox/ mmol de compuesto (Re et al., 1999).

El análisis de los datos mediante el procedimiento de diferencia mínima significativa (LSD) de Fisher, muestra que entre EIC y EIP existen diferencias estadísticamente significativas en el valor TEAC $(p<0.05)$; fue evidente una mayor capacidad de reducción (CRFC) y un mayor contenido antociánico para la muestra purificada (EIP); lo que evidencia la eficiencia del proceso de purificación mencionado anteriormente. Se ha establecido que muestras con una alta CRFC experimentan una fuerte actividad antioxidante (Rice Evans et al., 1996), sin embargo se observa que el valor TEAC del EIC es alrededor de 2.5 veces mayor que el del EIP (Tabla 1), este comportamiento sugiere que durante el proceso de purificación se eliminan compuestos (por ejemplo, carotenos, vitaminas, ácidos orgánicos, etc) que pueden ejercer algún efecto sinérgico en el extracto, ocasionando así una disminución de la actividad antioxidante, este comportamiento ya ha sido observado por Shi et al. (2014). De otro lado, un trabajo realizado por Bravo et al. (2016) muestra para el extracto metanólico del fruto de Monnina speciosa un valor TEAC de $0.0664 \mathrm{mmol}$ trolox $/ \mathrm{g}$, que es inferior al encontrado en este trabajo para el extracto metanólico (EIC) de Monnina Obtusifolia ( $8.5 \mathrm{mmol}$ trolox/g muestra).

Estadísticamente también se observó que entre las antocianinas 1: piranoantocianidina (vinilpiranopeonidin3-O- $\beta$-(acetil) glucopiranosil-O- $\alpha$-(succinil) ramnosi-do) y 3: Peonidin-3-O-(cumaroil) hexosa-O-(cumaroil, xidroxibenzoil) hexosa; ambas derivadas de la aglicona peonidina, existen diferencias significativas en el valor TEAC, lo que está en concordancia con lo publicado en la literatura, es decir que las piranoantocianinas son menos antioxidantes que las antocianinas (Sousa et al., 2013; 2016). En la tabla 1, también se puede observar que la antocianina 2: delfinidina-3-O- $\beta$-(fumaroil, malioil, propionil) glucopiranosil-O- $\alpha$-(fumaroil, butiroil, propionil) ramnosido; un derivado de delfinidina (tres grupos $\mathrm{OH}$ en $3^{\prime}, 4^{\prime}, 5^{\prime}$ ) es quien presenta la mayor actividad antioxidante (1.64 mmol trolox/g muestra), seguido por el compuesto 4: Cianidina-3-O-(malonil) glucopiranosil-O-(galoil, propionil) xilosido; un derivado de cianidina (dos grupos $\mathrm{OH}$ en 3' y 4').

Estos resultados de actividad antioxidante de los compuestos puros aislados de Monnina Obtusifolia nos llevan a la conclusión de que aquellas agliconas con solo un grupo $\mathrm{OH}$ en el anillo $\mathrm{B}\left(4^{\prime}-\mathrm{OH}\right)$ como las antocianinas 1 y 3 presentan menor actividad antioxidante comparado con compuestos que poseen agrupación 3',4' di-OH sustituidos como los derivados de delfinidina y cianidina (antocianinas 2 y 4 respectivamente) (Figura 2). Otros estudios han evidenciado la importancia de los grupos hidroxilos en la posición 3' y 4' del anillo B; sobre la mayor capacidad antioxidante de este tipo de moléculas (Rice-Evans et al., 1996). 
Adicionalmente fue evidente que todos los compuestos purificados de Ivilan son más antioxidantes que el ácido ascórbico, así que; los antocianos aislados del fruto poseen importantes propiedades antioxidantes. Algunos estudios mencionan que diversas frutas pueden constituir una buena fuente de compuestos polifenólicos capaces de impactar positivamente en la salud humana (He et al., 2010). Por esta razón hay un gran interés en la aplicación de este tipo de compuestos en alimentos y en la industria nutraceutica y farmacéutica. De hecho hay gran evidencia que los polifenoles tienen propiedades antioxidantes (Ramirez et al., 2015), anticancerígenos (Bontempo et al., 2015), tienen actividad antidiabética (Hong et al., 2013), contra la obesidad (Xie et al., 2018), previenen enfermedades cardiovasculares (Cassidy, 2018) y tienen potencial para prevenir la pérdida de memoria y enfermedades neurodegenerativas (Gutierres et al., 2014).

\section{CONCLUSIONES}

De los resultados mostrados, de su análisis y de su discusión, se pueden obtener las siguientes conclusiones con respecto a los polifenoles (antocianinas) aislados del fruto de Monnina Obtusifolia:

El contenido de antocianinas del fruto es de $95.3 \pm 0.6 \mathrm{mg} \mathrm{Cy-3-glu/} 100 \mathrm{~g}$ de fruto fresco.

Los análisis demuestran que las fracciones aisladas del fruto de Monnina Obtusifolia., por sus características antioxidantes (mayores a las del ácido ascórbico), pueden ser consideradas como una fuente importante de compuestos bioactivos para su uso en la industria.

Mediante la utilización de diferentes técnicas cromatográficas fue posible purificar las antocianinas mayoritarias del fruto de Ivilan, facilitando así su caracterización por HPLC-ESI-MS y ${ }^{1} \mathrm{H}-\mathrm{RMN}$. Se identificaron parcialmente: una piranoantocianina (compuesto 1), un derivado de la aglicona delfinidina (compuesto 2), un derivado de peonidina (compuesto 3 ) y otro de cianidina (compuesto 4).

La antocianina más antioxidante fue el derivado de delfinidina: delfinidina-3-O- $\beta$-(fumaroil, malioil, propionil) glucopiranosil-O- $\alpha$-(fumaroil, butiroil, propionil) ramnosido

Se estableció que las agliconas con solo un grupo $\mathrm{OH}$ en el anillo $\mathrm{B}\left(4^{\prime}-\mathrm{OH}\right)$, como el derivado de peonidina, presenta menor actividad antioxidante comparado con compuestos que poseen agrupación 3',4' di-OH sustituidos como los derivados de delfinidina y cianidina.

\section{AGRADECIMIENTOS}

Los autores agradecen el apoyo económico brindado por la Vicerrectoría de Investigaciones Postgrados y Relaciones Internacionales- VIPRI - UDENAR.

\section{REFERENCIAS}

Agrawal, P.K, NMR Spectroscopy in the Structural Elucidation of Oligosaccharides and Glycosides, doi: 10.1016/00319422(92)83678-R, Phytochemistry, 31(10), 3307-3330 (1992)

Andersen, Ø.M. у T. Fossen, Characterization of Anthocyanins by NMR, Current Protocols in Food Analytical Chemistry, 1-24, John Wiley \& Sons, New York, Estados Unidos (2003)

Bontempo, P., L. de Masi y otros nueve autores, Anticancer Activities of Anthocyanin Extract from Genotyped Solanum tuberosum, doi: 10.1016/j.jff.2015.09.063, J. Funct. Foods, 19, 584-593 (2015)

Bravo, K., F. Alzate y E. Osorio, Fruits of selected wild and cultivated Andean plants as sources of potential compounds with antioxidant and anti-aging activity, doi: 10.1016/j.indcrop.2015.12.074, Ind Crops Prod, 85, 341-352, (2016)

Bueno, J.M., P. Sáez-Plaza y otros cuatro autores, Analysis and Antioxidant Capacity of Anthocyanin Pigments, doi: 10.1080/10408347.2012.680343, Crit. Rev. Anal. Chem., 42(2), 126-151 (2012)

Cabrita, L. y Ø.M. Andersen, Anthocyanin Trisaccharides in Blue Berries of Vaccinium padifolium, doi: 10.1016/S03088146(99)00230-7, Food Chem., 69, 33-36 (2000)

Cassidy, A., Berry Anthocyanin Intake and Cardiovascular Health, Mol. Aspects Med., 61(6), 76-82 (2018)

Dehenhardt, A., H. Knapp y P. Winterhalter, Separation and Purification of Anthocyanins by High-Speed Countercurrent Chromatography and Screening for Antioxidant Activity, J. Agric. Food Chem., 48 (2), 338-343 (2000)

Flores, E., Extracción de Antioxidantes de las Bayas del Sauco (Sambucus nigra L. subsp. peruviana) con Ultrasonido, Microondas, Enzimas y Maceración para la obtención de Zumos Funcionales, doi: 10.4067/S0718-07642017000100012, Inf. Tecnol, 28(1), 121-132 (2017)

Flores, F.P., R.K. Singh y F. Kong, Anthocyanin Extraction, Microencapsulation, and Release Properties During in vitro Digestión, Food Rev Int., 32(1), 46-67 (2016) 
Giusti, M. y R.E. Wrolstad, Acylated Anthocyanins from Edible Sources and their Applications in Food Systems, Biochem. Eng. J., 14(3), 217-225 (2003)

Giusti, M.M., L.E. Rodriguez y R.E. Wrolstad, Molar Absortivity and Color Characteristics of Acylated and no-acylated Pelargonidin-based Anthocyanins, J. Agric. Food Chem., 47, 4631-4637 (1999)

Gutierres, J.M., F.B. Carvalho y otros diez autores, Neuroprotective Effect of Anthocyanins on Acetylcholinesterase Activity and Attenuation of Scopolamine-Induced Amnesia in Rats, Int. J. Dev. Neurosci., 33, 88-97 (2014)

He, J.A. y M. Giusti, Anthocyanins: Natural Colorants with Health Promoting Properties, Annu. Rev Food Sci. Technol., 1, 163-187 (2010)

Henke, H., Preparative Gel Chromatography on Sephadex LH-20, Journal Fur Praktische Chemie Chemiker-Zeitung, 338 (1), 291-295 (1996)

Hong, S.H., J.I. Heo y otros diez autores, Antidiabetic and Beta Cell-Protection Activities of Purple Corn Anthocyanins, Biomol Ther, 21, 284-289 (2013)

Hurtado, N.H. y M. Pérez, Identificación y Actividad Antioxidante de las Antocianinas Aisladas del Fruto de Capulí (Prunus serótina spp capulí (Cav) Mc. Vaug Cav), doi: 10.4067/S0718-07642014000400015, Inf. Tecnol, 25 (4), 131-140 (2014)

Kong, J.M., L. Chia, N. Goh y T. Chia, Analysis and Biological Activities of Anthocyanins, Phytochemistry, 69, 1939-1940 (2003)

Kuskoski, M. y A. Asuero, Actividad Antioxidante de Pigmentos Antocianicos, Ciencia y Tecnología de Alimentos, 24, 691693 (2004)

Lepore, L., N. Malafronte y otros cinco autores, Isolation and Structural Characterization of Glycosides from an Antiangiogenic Extract of Monnina obtusifolia H.B.K, doi: 10.1016/j.fitote.2010.08.018, Fitoterapia, 82(2), 178-183 (2011)

Mateus, N., A.M.S. Silva y otros tres autores, A New Class of Blue Anthocyanin-Derived Pigments Isolated from Red Wines, J. Agric. Food Chem., 51(7), 1919-1923 (2003)

Matsufuji, H., T. Otsuki y otros tres autores, Identification of Reaction Products of Acylated Anthocyanins from Red Radish with Peroxyl Radicals, J. Agric. Food Chem., 51, 3157-3161 (2003)

Menzies, I.J.L., W. Youard y otros cinco autores, Leaf Colour Polymorphisms: A Balance between Plant Defence and Photosynthesis, J. Ecol., 104, 104-113 (2016)

Re, R., N. Pellegrini y otros cuatro autores, Antioxidant Activity Applying an Improved ABTS Radical Cation Decolorization Assay, Free Radic. Biol. Med., 26 (9), 1231-1237 (1999)

Rentzsch, W., M. Schwarz y P. Winterhalter. Pyranoanthocyanins - an Overview on Structures, Occurrence, and Pathways of Formation, Trends Food Sci. Technol., 18(10), 526-534 (2007)

Rice Evans, C.A. y N.J. Miller, Antioxidant Activities of Flavonoids as Bioactive Components of Food, Biochem. Soc. Trans., 24(3), 790-795 (1996)

Rice-Evans, C.A., N.J. Miller y G. Papaganda, Structure Antioxidant Activity Relationships of Flavonoids and Phenolic Acids, Free Radic. Biol. Med., 20, 933-956 (1996)

$\mathrm{Shi}_{2}$ J., Y. Kakuda y D. Yeung, Antioxidative properties of lycopene and other carotenoids from tomatoes: synergistic effects, Biofactors, 21(1-4), 203-210 (2004)

Singleton, V.L., L. Orthofer y R.M. Lamuela-Raventós, Analysis of Total Phenols and Other Oxidation Substrates and Antioxidants by Means of Folin Ciocalteu Reagent, doi: 10.1016/S0076-6879(99)99017-1, Meth. Enzymol., 299 (1), $152-$ 178 (1999)

Sousa, A., P. Araújo y N. Mateus, Deoxyvitisins: A New Set of Pyrano-3-deoxyanthocyanidins, Tetrahedron Lett, 28, 47854788 (2013)

Sousa, A., P. Araújo y otros cuatro autores, Antioxidant and Antiproliferative Properties of 3-deoxyanthocyanidins, Food Chem, 192(1), 142-148 (2016)

Wang, Y., G. Luan y otros cinco autores, Subcritical Water extraction, UPLC-Triple-TOF/MS Analysis and Antioxidant Activity of Anthocyanins from Lycium Ruthenicum Murr, Food Chem., 249, 119-126 (2018)

Welch, C.R., Q. Wu y J.E. Simon, Recent Advances in Anthocyanin Analysis and Characterization, Curr. Anal Chem., 4(2), 75-101 (2008)

Wrolstad, R., Natural Food Colorants, 237-252, Marcel Dekker Inc, New York, Estados Unidos (2000)

Xie, L., H. Sua y otros tres autores, Recent Advances in Understanding the Anti-obesity Activity of Anthocyanins and their Biosynthesis in Microorganisms, Trends Food Sci. Technol., 72, 13-24 (2018)

Zhao, C.L., Y.Q. Yu y otros cinco autores, Stability-Increasing Effects of Anthocyanin Glycosyl Acylation, Food Chem, 214, 119-128 (2017) 